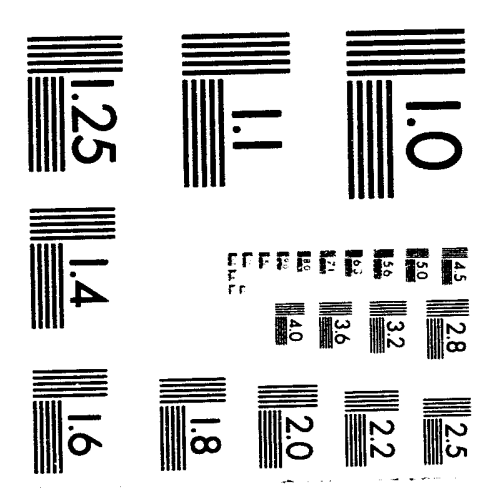



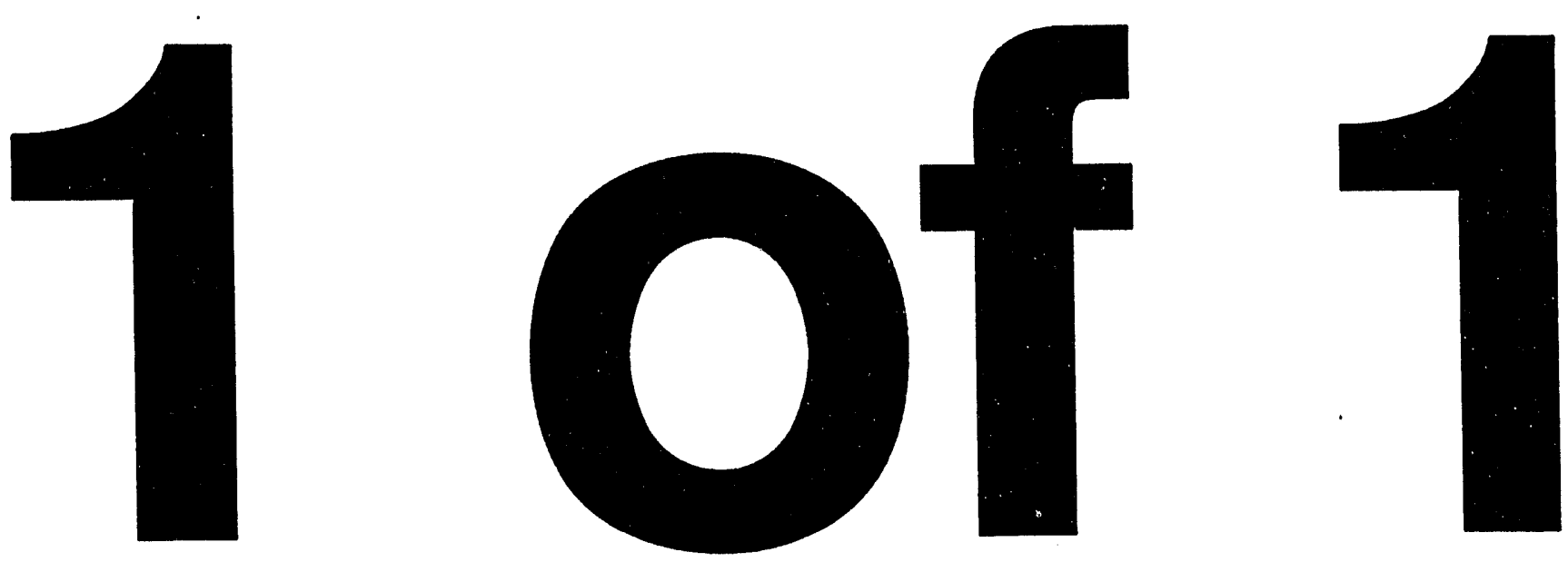


\section{CLUSTER RESEARCH IN THE FRAMEWORK OF THE THEORY INSTITUTE}

\section{J. Jellinek}

\section{Chemistry Division, Argonne National Laboratory, Argonne, IL 60439}

A new framework, The Theory Institute, for theoretical investigation of atomic clusters, was initiated in 1991 in the Chemistry Division. Its primary goal is to foster long-term collaborations with leading centers of theoretical studies of clusters around the world. The mode of the collaborations includes visits of theorists from different institutions (national and international) at Argonne for periods ranging from one to six months.

The collaboration with Professor S. F. Chekmarev (Siberian Branch, Academy of Sciences, Russia) focuses on formulating and applying novel optimal theoretical tools for studies of fundamental properties of clusters (or, more generally, $\mathrm{N}$-body systems), such as their stability, phases and phase changes. We have worked out a new, so-called microcanonical, Monte Carlo (MC) procedure. Circumventing the necessity of solving the dynamical equations of motion, it allows one to efficiently sample the configuration space - and, thus, the isomers and phases - of a cluster at a given total energy. We have also formulated three general and practical schemes for calculating the absolute density of states - one of the most fundamental characteristics of any physical system, be it an atom, molecule, cluster or bulk matter. Very little was known on how to obtain this quantity in the general case of anharmonic systems. Two of our approaches are based on molecular dynamics simulations, while the third capitalizes on the new microcanonical MC. Work is in progress on applying these approaches to specific clusters. We expect our schemes will find broad application in different areas of physics and chemistry.

The collaboration with Professors V. Bonacic-Koutecky and J. Koutecky (Free University, Berlin, Germany) and Professor P. Fantucci (University of Milan, Italy) is directed at producing an ab initio (as understood by chemists) molecular dynamics (MD) code for studying metal cluster and metal cluster-molecule systems. The goal is an accurate scheme that does not invoke model potentials. The Argonne compenent of the collaboration is responsible for the nuclear dynamics, while the Berlin-Milan group is working on the electronic structure. A preliminary version of the code is being tested on $\mathrm{Li}_{4}$ and $\mathrm{Li}_{6}$ clusters. Because of the extremely high computational demands of the problem, the current effort focuses on codes optimization, including parallelization. It is planned that the ab initio MD program will be adapted to and installed on Argonne's Delta Touchstone multiprocessor machine.

We continue our collaboration with Dr. I. Garzon (Ensenada Laboratory, Institute of Physics, National Autonomous University of Mexico) on studies of structural and dynamical properties of metal clusters using semiempirical potentials. An extensive comparative study of nickel and gold clusters has been carried out. The most stable structures of the same-size clusters of these two metals are very different. Some nickel clusters, e.g., Ni13, show an unexpectedly small reduction in their melting temperature as compared to the melting temperature of the bulk. Nickel clusters of sizes, which form complete icosahedra plus an extra atom, exhibit an unknown earlier phenomenon of "premelting"; the latter is a local melting taking place at temperatures that are much lower than the corresponding actual melting of the cluster. The reduction in the melting temperature with cluster size is larger for gold than for nickel. Our results indicate that for smaller gold clusters, this reduction has the tendency to stabilize around $50 \%$ of the bulk melting temperature. Recent experiments at Purdue University support this finding.

The Institute offers a flexible and cost-efficient framework for collaborative theoretical research in the rapidly developing field of atomic clusters. Initiated during relatively short visits of scientists to Argonne, this research then continues in the respective home institutions, with possible periodic revisits of the collaborators. We plan to expand the activity of the Theory Institute to include other areas of research in the Chemistry Division.
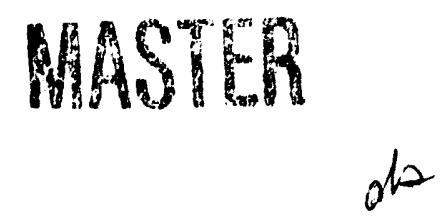

DISTMaUHDN OF THIS DOCUMENT IS UNLIRAITEA 


\section{CLUSTER RESEARCH IN THE FRAMEWORK OF THE THEORY INSTITUTE}

\section{J. Jellinek}

\section{Chemistry Division, Argonne National Laboratory, Argonne, IL 60439}

A new framework, The Theory Institute, for theoretical investigation of atomic clusters, was initiated in 1991 in the Chemistry Division. The primary goal of the Institute is to foster long-term collaborations with leading centers of theoretical studies of clusters worldwide. The mode of the collaborations includes visits of theorists from different institutions (international and national) to Argonne for periods ranging from one to six months.

Professor S. F. Chekmarev (Institute of Thermophysics, Siberian Branch of the Academy of Sciences, Novosibirsk, Russia) visited Argonne for 3.5 months. This collaboration focuses on formulating and applying novel optimal (efficient and adequate) theoretical tools for studies of fundamental properties of clusters (or, more generally, N-body systems), such as their stability, phases and phase changes. We have worked out a new, so-called microcanonical, Monte Carlo (MC) procedure for Hamiltonian systems. It is based on an analytical expression for the momentum-space volume corresponding to a given configuration of an $\mathrm{N}$-body system with a specified total energy. By circumventing the necessity to solve the dynamical equations of motion, the microcanonical MC accomplishes a very efficient ("importance") sampling of the corifiguration space - and, thus, of the isomers and phases - available to a cluster at a fixed total energy. The traditional canonical (Metropolis) MC is appropriate only for systems at a fixed temperature, i.e., with a Boltzmann distribution of energies. We have written an algorithm in which both the microcanonical and the canonical MC sampling procedures are performed in the course of the same run.

Another aspect of this collaboration is devoted to the problem of calculating the density of states - one of the most fundamental characteristics of any physical system, be it an atom, molecule, cluster or bulk matter. In order to understand and to characterize some important phenomena one has to know the absolute (normalized), as opposed to the relative, density of 
states. In the context of cluster studies, the absolute density of states is needed for exploring the question of why the individual cluster features evolve with cluster size in the way they do. The phenomenon of cluster evaporation, as well as that of cluster growth, are other examples of processes that cannot be described accurately without the knowledge of the absolute density of state. The difficulty is that this quantity can be obtained analytically only for harmonic systems, and very little has been known on how to calculate it in the general case. We have formulated three new approaches for calculating the absolute density of states of arbitrary systems numerically. Two of these are based on molecular dynamics simulations, and one on the above-mentioned microcanonical MC. Work is in progress on applying these approaches to specific clusters. We expect that they will find broad application in different areas of physics and chemistry.

The collaboration with Professors V. Bonacic-Koutecky and J. Koutecky (Free University, Beriin, Germany) and Professor P. Fantucci (University of Milan, Italy) is directed at producing an ab initio (as understood by chemists) molecular dynamics code for studying metal clusters and metal cluster-molecule systems. The goal is an accurate description that does not invoke model potentials. We (Argonne) are responsible for the nuclear dynamics part, while the Berlin-Milan group is working on the electronic structure component. Because of the extremely high computational demands of the problem, the current effort focuses on code optimization and "tuning". A preliminary version is being tested on $\mathrm{Li}_{4}$ and $\mathrm{Li}_{6}$ clusters. Work is in progress on parallelizing the codes, and we plan to install them on Argonne's Delta Touchstone multiprocessor machine. Professors V. Bonacic-Koutecky and P. Fantucci will each spend a month at Argonne in May-June, 1992.

Dr. I. L. Garzon (Ensenada Laboratory, Institute of Physics, National Autonomous University of Mexico) is currently at Argonne on a three-month visit. We continue our investigation of structural and dynamical properties of nickel and gold clusters using a semiempirical potential. Our main findings can be summarized as follows. The most stable configurations of nickel clusters are different from those of gold clusters of the same size. Interestingly, these configurations for nickel are closer to the corresponding minimum energy structures of Van der 
Waals (e.g., noble gas) clusters. A solid-to-liquid like transition takes place in clusters at temperatures below the bulk melting temperature, which is in accord with earlier studies of the melting behavior of noble gas and alkali halide clusters. A novel feature, however, is the unusually high degree of stability (resisitivity to melting) of nickel clusters of a certain size, for example $\mathrm{Ni}_{13}$. A new element in the melting mechanism, which we called "premelting", has been found in $\mathrm{Ni}_{14}$ and $\mathrm{Ni}_{20}$. Premelting is a localized melting of part of the cluster taking place at temperatures much lower than the actual melting. Premelting is characteristic of nickel clusters of those sizes that form complete icosahedra with an extra atom. Gold clusters do not exhibit the phenomenon of premelting. Our calculations indicate that the reduction in the melting temperature with cluster size is larger in gold than in nickel. For smaller gold clusters the reduction, however, seems to stabilize in the vicinity of $50 \%$ of the bulk gold melting temperature. Recent experiments at Purdue University by Professor R. Reifenberger in R. Andres' group support this finding. Work on other aspects of the dynamics of metal clusters, such as surface melting in larger size clusters, is in progress.

The benefits of the Theory Institute are that it provides a flexible and cost-efficient framework for collaborative theoretical research in the rapidly developing, inherently interdisciplinary field of atomic clusters. Collaborations of variable scope and duration with experts in the areas of current interest are established through their relatively short visits to Argonne. The joint research then continues in the respective home institutions with possible periodic revisits of the collaborators to Argonne. We plan to use the accumulated experience to expand the activity of the Theory Institute to other areas of research in the Chemistry Division.

\section{DISCLAIMER}

This report was prepared as an account of work sponsored by an agency of the United States Theither the United States Government nor any agency thereof, nor any of their Government. Neither the United States Government nor any agency the liability or responsiemployees, makes any warranty, express or implied, or assumes any legal or bility for the accuracy, completeness, or usefulness of any information, appaty owned rights. Referprocess disclosed, or represents that its use would not inf or service by trade name, trademark, ence herein to any specific commercial product, process, or service by its endorsement, recommanufacturer, or otherwise does not necessarily constitute or imply its endorsement, recommendation, or favoring by the United States Government or any agency the and opinions of authors expressed herein do not

United States Government or any agency thereof. 

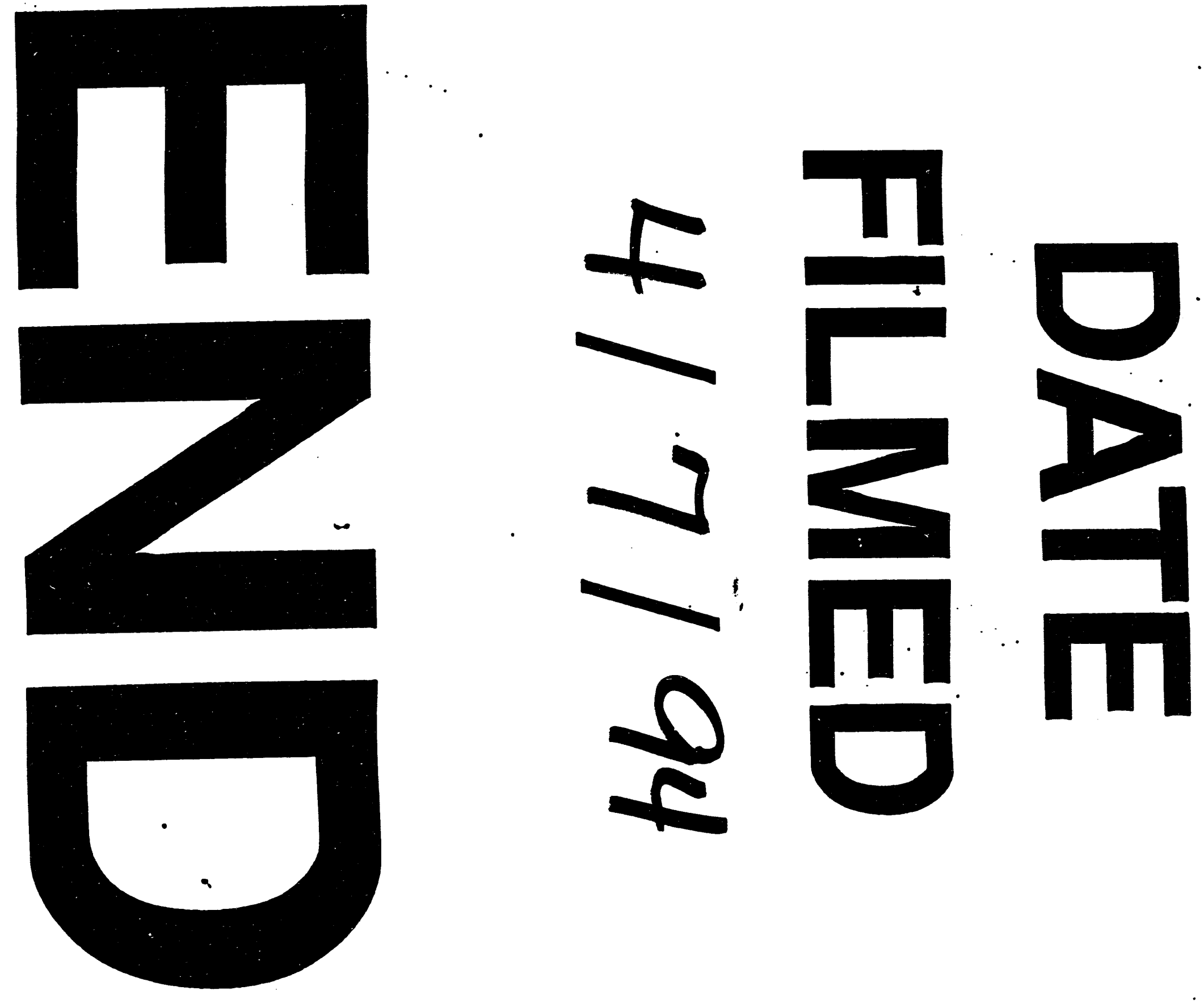
.

$-$

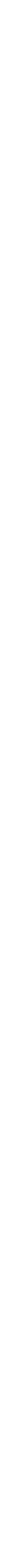
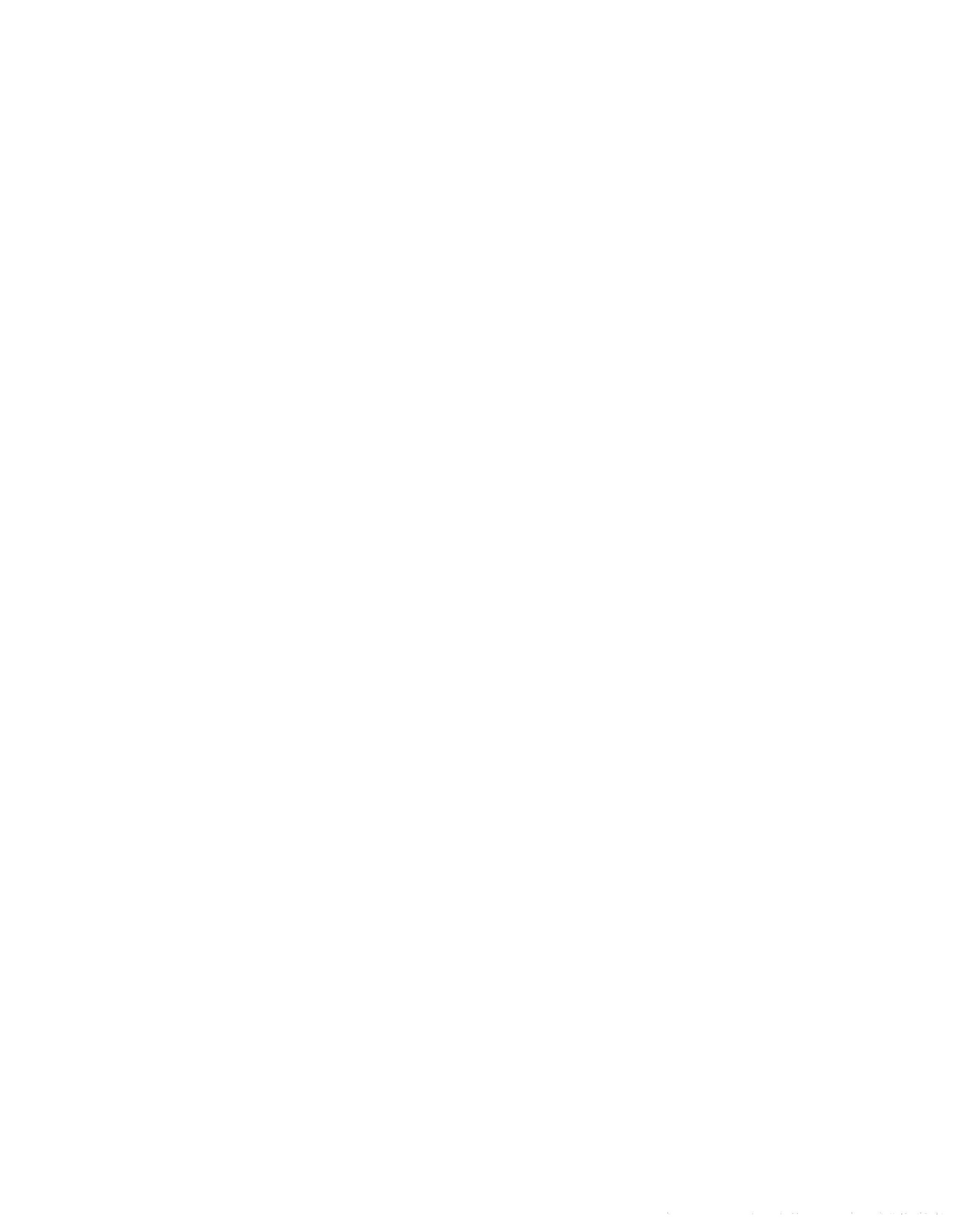